\section{Mikrobiom hilft heilen}

In zwei Publikationen fanden sich 2013 Hinweise darauf, dass die Darmflora, insbesondere grampositive Bakterien, möglicherweise einen wichtigen Beitrag zur Wirksamkeit von Chemotherapien mit Cyclophosphamid oder Platinsalzen leistet [Viaud S et al. Science. 2013; 342(6161):971-6; Iida N et al. Science. 2013;342(6161):967-70]. Diesen tierexperimentellen Ergebnissen fügten Kölner Forscher jetzt erste Hinweise auch beim Menschen hinzu.

Dazu untersuchten sie retrospektiv Daten von 800 Patienten mit chronischer lymphatischer Leukämie (CLL) der CLL8-Studie, die in der Erstlinie Fludarabin/Cyclophosphamid alleine (FC) oder zusammen mit Rituximab (FCR) erhalten hatten, sowie von Patienten mit rezidivierten Lymphomen aus einer Kölner Kohorte, die mit zwei Zyklen von Dexamethason, Cytarabin und Cisplatin (DHAP) alleine oder plus Rituximab (RDHAP) behandelt worden waren.

Weil die tierexperimentellen Ergebnisse vor allem auf eine Rolle grampositiver Bakterien hinwiesen, wurde in der Studie der Einfluss primär auf diese Keime gerichteter Antibiotika wie Vancomycin, Teicoplanin, Linezolid und Dap- tomycin auf das Ansprechen und Überleben untersucht. Dabei zeigte sich bei den Patienten der CLL8-Studie, dass die Gabe solcher Antibiotika ein signifikanter, von anderen Faktoren unabhängiger Risikofaktor für das Gesamtüberleben zu sein scheint. Das Ansprechen war klar schlechter als ohne Antibiotika mit Wirkung gegen grampositive Bakterien (Komplettremission: 22,9 vs. $33,8 \%$, partielle Remission 51,4 vs. $56,4 \%$ und fehlendes Ansprechen 25,7 vs. 9,8\%). Die 3-Jahres-Überlebensrate lag bei den 755 Patienten, die keine oder nicht für gram-positive Bakterien relevante Antibiotika erhalten hatten, bei $57,9 \%$, bei den 45 mit relevanten Antibiotika behandelten Patienten dagegen bei 32,8 ( $p<0,001)$. Der Vorteil blieb in einer Subgruppenanalyse auch bei verschiedenen CLL-Risikogruppen signifikant, z. B. bei Patienten mit del17p-Mutation, hohem s $\beta 2$-Mikroglobulin-Wert oder weniger als sechs Therapiezyklen.

Bei der Kölner Kohorte von Patienten mit rezidivierten Lymphomen schien die Assoziation von Antibiotikagabe mit Wirkung gegen grampositive Bakterien und eine ungünstigere Prognose sogar noch stärker (3-Jahres-OS: 15,8 vs. 1,0\%; $\mathrm{p}=0,001)$. Wiederum fand sie sich auch

\title{
CLL: FCR-Dosisreduktion vermeiden
}

Die Wirksamkeit der Kombination von Fludarabin, Cyclophosphamid und Rituximab (FCR) als Erstlinienbehandlung bei chronischer lymphatischer Leukämie (CLL) ist abhängig davon, ob $80 \%$ und mehr der Zieldosis von R oder FC erreicht werden kann. Das bestätigen gepoolte Daten aus zwei Studien der Deutschen CLL-Studiengruppe (CLL8 und CLL10). Von 635 behandelten Patienten hätten 428 mindestens $80 \%$ der geplanten Dosis erhalten, 253 nicht, berichtete Barbara Eichhorst, Köln. Das wirkte sich signifikant sowohl auf das mediane progressionsfreie Überleben (PFS, 64 vs. 45,2 Monate; $p<0,001$ ), als auch das mediane Gesamtüberleben (OS, nicht erreicht vs. 90,2 Monate; $p<0,001$ ) aus. Als Risikofaktoren für eine Dosisreduktion über $20 \%$ hinaus wurden identifiziert: Alter über 60 Jahre, Binet-Stadium C und erhöhte Serum- $\beta 2$ Mikroglobulin-Werte (> 3,5 mg/l). Diese Parameter könnten laut Eichhorst zukünftig eventuell helfen zu entscheiden, wer eine weniger toxische Therapie erhalten sollte.

Bendamustin/Rituximab (BR) mit Lenalidomid zu kombinieren, ist da keine Alternative und kann laut Natali Pflug, Köln, nicht empfohlen werden. Eine entsprechende Phase-I/ II-Studie (CLL2P) sowohl mit vorbehandelten als auch nicht vorbehandelten Patienten mit CLL musste abgebrochen werden. Die Toxizität war hoch: Unter BR plus Lenalidomid entwickelten $93 \%$ der vorbehandelten Patienten Grad-3/4-Nebenwirkungen und $100 \%$ der nicht vorbehandelten Patienten im Vergleich zu 51,3 und 52,1\% der Patienten unter BR. Entsprechend hoch war die Rate an Therapieabbrüchen sowie Dosisreduktionen und auch die Effektivitätsdaten waren enttäuschend (Gesamtansprechrate: $47,1 \%$ bei vorbehandelten, $60 \%$ bei nicht vorbehandelten Patienten vs. 59 und $88 \%$ bei BR). Die Rekrutierung für die Erstlinienkohorte gestaltete sich schwierig - auch aufgrund der zunehmend als Alternative verfügbaren neuen Substanzen. Friederike Klein

bei unterschiedlichen Subgruppenanalysen, allerdings war die Kohorte mit insgesamt 122 Patienten für solche Analysen relativ klein.

\section{Nur Assoziation, noch kein kausaler Zusammenhang!}

Maria J. G. T. Vehreschild, Köln, betonte, dass es sich hier nur um Assoziationen handele, die keine Rückschlüsse auf kausale Zusammenhänge zuließen. Konsequenzen in der klinischen Praxis ergeben sich daraus erst einmal nicht, wohl aber Ansätze, die Rolle des Mikrobioms bei der Wirksamkeit der Chemotherapie beim Menschen weiter zu untersuchen.

Den 2013 publizierten tierexperimentellen Befunden zufolge führt die Beeinträchtigung der Mucosabarriere im Darm durch die Chemotherapie dazu, dass Bakterien von dort transloziert werden und einerseits in der Milz TH17Zellen primen sowie andererseits myeloide Zellen konditionieren. Diese potenziell für die Tumorreduktion relevanten Kommunikationswege werden nach dieser Hypothese durch die Antibiotikagabe blockiert.

\section{Immuntherapie bei Nierenkrebs wandelt sich}

Die Immuntherapie mithilfe von Zytokinen wie Interferon und Interleukin-2 hat seit mehreren Jahren dazu beigetragen, die Prognose von Patienten mit einem Nierenzellkarzinom (RCC, ,renal cell cancer") zu verbessern. Es wurde damit ein medianes Überleben von $13 \mathrm{Mo-}$ naten erreicht, wie Viktor Grünwald, Hannover, erinnerte. Noch bessere Erfolge lassen sich mit der modernen Immuntherapie erzielen, in der Checkpointhemmer wie Nivolumab verwendet werden. Median sind mit derartigen Immuntherapeutika bis zu 29 Monate Überleben zu erreichen.

Wie sehr sich die Prognose verbessert hat, geht unter anderem aus der aktuellen Checkmate-25-Studie hervor, wie Grünwald berichtete. In der großen Studie mit mehr als 820 vorbehandelten Patienten, die an einem klarzelligen RCC erkrankt waren, wurde die Wirksamkeit - gemessen am Parameter Gesamtüberleben - von Nivolumab im Vergleich zum mTOR(„mechanistic Target 